\title{
A new noise detected in the ocean
}

\author{
Angel Guerra*đ, Xavier Martinell ${ }^{\dagger}$, Angel F. González*, Michael Vecchione”, \\ Joaquin Gracia ${ }^{\jmath}$ and Jordi Martinell ${ }^{\S}$
}

\author{
*Instituto de Investigaciones Marinas (CSIC), Vigo, Spain. ${ }^{*}$ Mediterráneo Servicios Marinos S.L., Ibiza, Spain; *NMFS National \\ Systematics Laboratory, National Museum of Natural History, Washington DC, USA. `Marexi Corporation, Vigo, Spain. ${ }^{\S}$ Facultat de
}

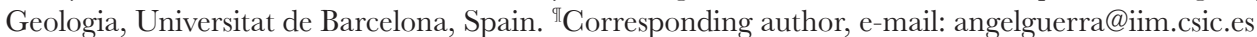

\begin{abstract}
Many observers have noted that the sea is full of loud sounds, both ongoing and episodic. Among the many sources of natural ambient noise are wave action, physical process such as undersea earthquakes, and biological activities of shrimps, fishes, dolphins and whales. Despite interest by acoustics experts, sound production by cephalopods has been reported only twice, both involving squid. The 'faint poppings' produced were thought to result from fluttering of the thin external lips of the squid's funnel while water is being expelled through it. Otherwise, no information is available on cephalopod sounds. Here we present a noise produced by a stressed common octopus. The event was filmed and recorded in the wild. The hypothesis we offer to explain how this sound was produced is cavitation, which has been documented in several biological systems. In our case, the water expelled through the funnel may have created a jet with velocity so high that the turbulent pressure dropped locally below the vapour pressure of the water. Sea water contains gas microbubbles, which will grow in size when they are entrained in the region of low pressure. Subsequently, the bubbles collapse violently when pressure rises again. The sound produced by the octopus is like a gunshot, and distinct lights observed at the same time contradict the existence of a simple pressure wave and point to the possible presence of gas-bubbles, which would change the light intensity by reflection and refraction of the sunlight. This behaviour seems to be a defensive strategy to escape from vibration-sensitive predators.
\end{abstract}

We report that an octopus produced a brief, loud sound. This event, the first such observation in cephalopods, occurred while a two kilogram wild male of the common octopus (Octopus vulgaris) was filmed by a SCUBA diver. This happened on 1 July 2006 in the Balearic Islands (Western Mediterranean) at $12 \mathrm{~m}$ depth (water temperature: $25^{\circ} \mathrm{C}$; salinity: 35.8 ). The camera used was a Sony DSR PD150P and the film TDK DV quality SP. Neither autofocus nor flash was used during filming. The frame rate was $24 \mathrm{fps}$. The camera was provided with a built-in microphone, Electret condenser, operating in 'mono' mode.

The observer found the octopus while it was being attacked by a grouper that escaped when the diver approached. The observer then pursued and filmed the octopus. It went down towards the bottom and released ink before hiding underneath Posidonia oceanica plants (Figure 1A). While hidden, the animal again released ink. Also observed was a clearly distinguishable intense red color on the ventral side of the octopus (Figure 1B). Simultaneously, a strident shot-like sound of 135 milliseconds duration was produced by the animal (Figure 1C). With the equipment used, the sound recorder reached a peak of $24 \mathrm{KHz}$. Afterwards, a distinct light was observed outside the shaded area in the zone affected by the sunlight (Figure 1B). During and after the recording the animal adopted a defensive posture (Hanlon \& Messenger, 1996).

Sound production by cephalopods has been reported only twice, both involving squids (Nishimura, 1961; Iversen et al., 1963). These 'faint poppings', with frequencies ranging from 300 to 2400 $\mathrm{Hz}$, were thought to result from accidental fluttering of the thin external lips of the funnel while water was being expelled through it (Iversen et al., 1963).

We are not certain of the source of the sound produced by our octopus. However, we discarded in this case the fluttering hypothesis because a sound produced by vibration has different characteristics than that described herein. In this case, the sound is like a shot, and the unusual light observed contradicts the existence of a simple pressure wave. These observations point to the possible presence of gas-bubbles, which would change the light intensity by reflection and refraction of the sunlight. The hypothesis we offer to explain how this sound was produced is cavitation. The process could have been produced by an extraordinarily strong and rapid contraction of the mantle muscles once the octopus sensed extreme danger. The water expelled through the funnel could have created a jet with velocity so high that the turbulent pressure dropped locally below the vapour pressure of the water. Sea water contains gas microbubbles, which grow in size when they are entrained in a region of low pressure. Subsequently, the bubbles collapse violently when pressure rises again. That process is the most convincing explanation of the intense red color on the ventral side of the octopus and the distinct light observed outside the shaded area, which seems to be produced by the incidence of the solar light on cavitation bubbles.

Cavitation has been documented in several biological systems (Smith, 1991; Verlius et al., 2000). However, the most dynamic example of biological cavitation is found in snapping shrimp under controlled conditions. The sound produced by Alpheus heterochaelis with its snapper claw by cavitation results from powerful strikes of their raptorial appendages (Versluis et al., 2000). The extreme speed of underwater strikes by a mantis shrimp (Odontadactylus scyllurus) similarly causes cavitation (Patek \& Caldwell, 2005). The hard beak of the octopus should be also mentioned as a possible sound producer. The sound could have originated from the collapse of a cavitation bubble generated by a fast water jet resulting from the rapid closure of the lower and upper mandible, in a manner similar to that in the snapping shrimp claw.

If cavitation is causing noise in our octopus, an important question is whether this is an intentional behaviour of the animal. In other words, does the octopus have a use for the noise or is it an accidental by-product of hydrodynamics? In either case, noise production could be a disadvantage if a nearby predator is capable 

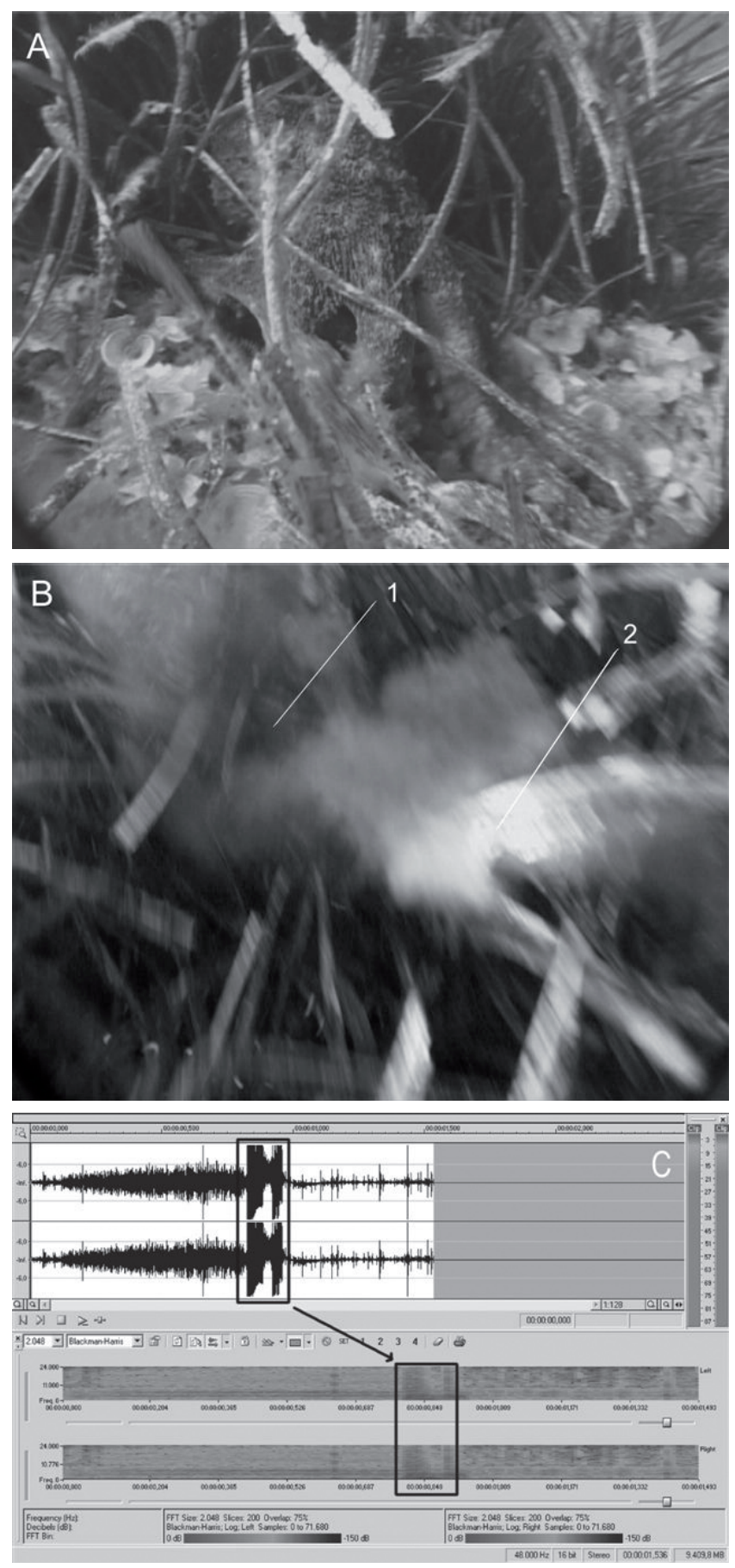

Figure 1. (A) The common octopus escaping from the diver and hiding underneath Posidonia oceanica plants; (B) a clearly distinguishable intense red color (1) was observed in the ventral area of the octopus. Afterwards, a distinct light (2) was observed outside the shaded area in the zone affected by the sunlight; $(\mathrm{C})$ simultaneously, a brief and loud shot-like sound was produced by the animal (arrow). of detecting the noise. There is therefore a selective advantage in being able to control the noise and only allow it to occur if really necessary. The most plausible explanation is, as seen in the footage, a defensive behaviour to escape from the predators. This would be produced by a rapid defensive mechanism that needs a large amount of energy. For this reason, such actions should be quite unusual and employed only under extreme circumstances.

The paper was reviewed by Dr Ian K. Bartol (Old Dominion University) who commented on several hydrodynamic aspects and improved a previous version. We would like to thank also Mr. Marcos Villafin for technical assistance.

\section{REFERENCES}

Hanlon, R.T. \& Messenger, J.B., 1996. Cephalopod behaviour. Cambridge: Cambridge University Press.

Iversen, R.T.S., Perkins, P.J. \& Dionne, R.P., 1963. An indication of underwater sound production by squid. Nature, London, 199, 250-51.

Nishimura, M., 1961. Frequency characteristics of sea noise and fish sound. Technical Reports of the Fisheries Boat Laboratory, Ministry of Agriculture for Fapan, Tokyo, 15, 111-118.

Patek, S.L \& Caldwell, R.L., 2005. Extreme impact and cavitation forces of a biological hammer: strike forces of the peacock mantis shrimp Odontadactylus scyllarus. Fournal of Experimental Biology, 208, 3655-3664

Versluis, M., Smihtz, B. von der Heydt, A. \& Lohse, D., 2000. How snapping shrimp snap: through cavitating bubbles, Science, New York, 289, 2114-2117.

Smith, A.M., 1991. Negative pressure generated by octopus suckers: a study of the tensile strength of water in nature. Fournal of Experimental Biology, 157, 257-271. 